\title{
PDE Methods for the Maximum Drawdown
}

\author{
Libor Pospisil, Jan Vecer \\ Columbia University, Department of Statistics, New York, NY 10027, USA
}

April 1, 2008

\begin{abstract}
Maximum drawdown is a risk measure that plays an important role in portfolio management. In this paper, we address the question of computing the expected value of the maximum drawdown using a partial differential equation (PDE) approach. First, we derive a two-dimensional convection-diffusion pricing equation for the maximum drawdown in the Black-Scholes framework. Due to the properties of the maximum drawdown, this equation has a nonstandard boundary condition. We apply an alternating direction implicit method to solve the equation numerically. We also discuss stability and convergence of the numerical method.
\end{abstract}

\section{Introduction}

Maximum drawdown of an asset is defined as the largest drop of the asset price within a certain time period. Maximum drawdown can be viewed as a contingent claim that can be priced and hedged using the standard risk-neutral valuation techniques. Moreover, the replication of payoffs that depend on the maximum drawdown can serve as insurance against adverse movements of the asset price during a market turmoil. In this paper, we focus on computing the expected value of the maximum drawdown for period $[0, T]$, a value that can be viewed as the price of a forward on the maximum drawdown. This expected value does not have a known analytical expression, therefore we develop a numerical method for pricing the forward.

We first present the derivation of a partial differential equation for the forward value in the Black-Scholes model. The equation has three spatial dimensions. We show that one can use a linear scaling property of the forward to transform the equation into a two-dimensional convection-diffusion equation with constant coefficients on a rectangular domain. Subsequently, we apply an alternating direction implicit method to find a numerical solution to the equation. There are several issues we must pay attention to. First, the cross-derivative term in the equation makes the diffusion matrix singular. Thus the equation is not parabolic in the strict sense. Second, the value function at the time of maturity does not satisfy a boundary condition of the equation. Third, one of the boundary conditions is of neither Dirichlet nor Neumann type. Finally, we analyze stability and convergence of the numerical method.

A contract on the maximum drawdown can be used as financial insurance in the following way. Many investors want to be exposed to a market while being protected from the adverse downturns of the market. Insurance is typically provided by buying out-of-the-money put options. However, the market may rally before it drops down. Therefore, the insurance provided by the put option might not come in effect even in the midst of a clear market crisis.

Figure 1 shows a simulated joint distribution of the S\&P 500 and its maximum drawdown for 3 month period in the future. We used closing values from March 20, 2008 (index value 1329.5, volatility 19\% from the historical data). Notice that some significant drawdowns occur when the value of the index is above the initial starting level of 1329.5 .

Maximum drawdown is also important for directional traders. In particular, momentum traders believe that current trends will continue, which implies that the realized drawdown, drawup, or range are believed 


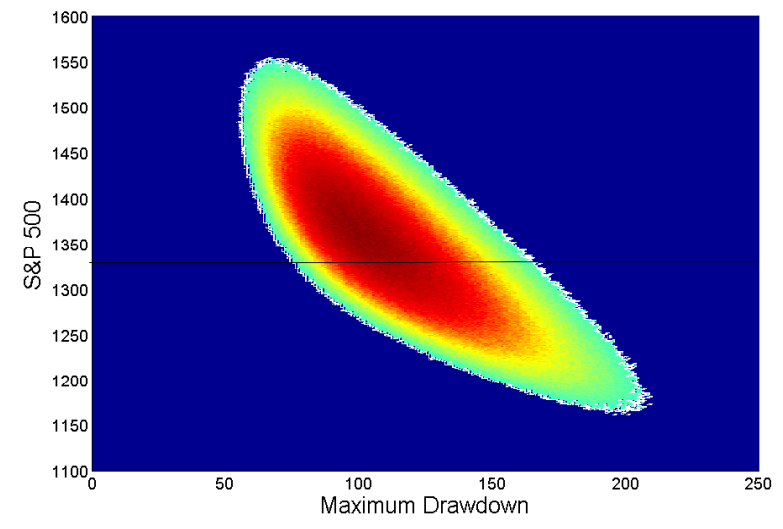

Figure 1: Simulated 3 month joint distribution of the S\&P 500 index and its maximum drawdown. We use values from March 20, 2008 (index value 1329.5, volatility 19\% from the historical data, drift 6\%). The outcomes above the line will not be protected by the out of the money put options since the final value of the index will be above the initial value of the index. However, some of these scenarios exhibit quite significant drawdowns.

to end up higher than expected. Contrarian traders believe the opposite, namely that trends will revert. In effect, contrarian traders believe that the realized drawdown, drawup, or range will be smaller than expected. Traditionally, the trends have been determined mainly from a regression analysis of the market data. Replicating contracts on the maximum drawdown can provide traders with additional tools to construct momentum trading strategies.

Maximum drawdown has been previously studied in the literature. A formula for the expected maximum drawdown of a drifted Brownian motion was derived in Magdon-Ismail et al. (2004). However, the formula contains an infinite sum of integrals without an analytical solution. Salminen and Vallois (2007) calculated the joint distribution of the maximum drawdown and the maximum drawup of a Brownian motion up to an independent exponential time.

Several authors discussed the use of maximum drawdowns in finance. Vaz de Melo Mendes and Ratton Brandi (2004) proposed the maximum drawdown as a risk measure. Grossman and Zhou (1993), Cvitanic and Karatzas (1995), and Chekhlov et al. (2005), addressed the problem of portfolio optimization with drawdown constraints. Vecer (2006) proposed that forwards and options on the maximum drawdown can be priced and hedged as derivative contracts. Pospisil and Vecer (2008) derived a probabilistic representation for the hedge of a forward on the maximum drawdown.

The alternating direction approach for solving multi-dimensional parabolic equations has been described in Strikwerda (1989) and Duffy (2006). The particular finite difference schemes we use in this paper were introduced in Peaceman and Rachford (1955) and Douglas and Rachford (1956). The stability and the convergence of alternating direction methods were analyzed, for example, in Hout and Welfert (2007) and Hundsdorfer and Verwer (1989).

This paper is organized as follows: In Section 2, we derive the two-dimensional partial differential equation for a forward on the maximum drawdown. In Section 3 and Section 4, we propose a numerical method for solving the equation. Then we discuss the stability and the convergence of the method, and carry out several numerical experiments. Section 5 contains concluding remarks. 


\section{Forward on the Maximum Drawdown}

In this section, we define a forward on the maximum drawdown in the Black-Scholes model. We show that its value function satisfies a three-dimensional partial differential equation that can be reduced to a two-dimensional convection-diffusion equation.

Throughout this paper, we will use the following notation. Let $S_{t}$ be the value of an asset at time $t$. Its running maximum $M_{t}$ is given by:

$$
M_{t}=\max _{u \in[0, t]} S_{u}
$$

Maximum drawdown $M D D_{t}$ is defined as the largest drop of the asset price from the running maximum up to time $t$ :

$$
M D D_{t}=\max _{u \in[0, t]}\left(M_{u}-S_{u}\right)
$$

Suppose that the asset value $S_{t}$ evolves according to a geometric Brownian motion under the risk-neutral measure $\mathbb{Q}$ :

$$
d S_{t}=r S_{t} d t+\sigma S_{t} d W_{t}, \quad t \geq 0
$$

where $r$ denotes a risk-free interest rate and $\sigma$ the asset volatility. Process $W$ is a Brownian motion under $\mathbb{Q}$. Let $\left(\mathcal{F}_{t}\right)_{t \geq 0}$ be the $\sigma$-field generated by $W$.

We define a forward on the maximum drawdown as a contract whose holder receives amount $M D D_{T}$ at time $T$. The standard no-arbitrage argument implies that its value at time $t, V_{t}$, is given by the conditional expectation under the risk-neutral measure $\mathbb{Q}$ :

$$
V_{t}=e^{-r(T-t)} \mathbb{E}\left[M D D_{T} \mid \mathcal{F}_{t}\right] .
$$

Since $S_{t}$ is a geometric Brownian motion, the three-dimensional process $\left(S_{t}, M_{t}, M D D_{t}\right)_{0 \leq t \leq T}$ has the Markov property and the distribution of $M D D_{T}$ depends on the information contained in $\mathcal{F}_{t}$ only through $\left(S_{t}, M_{t}, M D D_{t}\right)$. As a result, there is a function $v$, such that:

$$
V_{t}=v\left(t, S_{t}, M_{t}, M D D_{t}\right)=e^{-r(T-t)} \mathbb{E}\left[M D D_{T} \mid S_{t}, M_{t}, M D D_{t}\right]
$$

We are using a similar argument to that presented in Shreve (2004) for lookback options (see page 308). Note that function $v(t, s, m, m d d)$ is defined on the following domain:

$$
0 \leq t \leq T, \quad 0 \leq s<\infty, \quad s \leq m<\infty, \quad m-s \leq m d d<m .
$$

According to (2), the discounted value of the forward, $e^{-r t} V_{t}$, is a $\mathbb{Q}$-martingale. We use this fact in the following proposition, where we derive an equation for function $v$, including boundary conditions. Let us denote the partial derivatives of $v(t, s, m, m d d)$ as $v_{t}, v_{s}, v_{s s}, v_{m}$, and $v_{m d d}$.

Proposition 2.1 The value function of a forward on the maximum drawdown,

$$
v\left(t, S_{t}, M_{t}, M D D_{t}\right)=e^{-r(T-t)} \mathbb{E}\left[M D D_{T} \mid S_{t}, M_{t}, M D D_{t}\right]
$$

satisfies the following partial differential equation:

$$
\begin{gathered}
v_{t}(t, s, m, m d d)+r s v_{s}(t, s, m, m d d)+\frac{1}{2} \sigma^{2} s^{2} v_{s s}(t, s, m, m d d)=r v(t, s, m, m d d) \\
\text { on }(0, T) \times\{(s, m, m d d) ; 0<s<m \& m-s<m d d<m\} .
\end{gathered}
$$


with the terminal and the boundary conditions given by:

$$
\begin{aligned}
v(T, s, m, m d d) & =m d d, & & \\
v(t, s, m, m d d) & =e^{-r(T-t)} m d d & & \text { if } s=0 \text { or } m=m d d, \quad t \in[0, T), \\
v_{m}(t, s, m, m d d) & =0 & & \text { if } m=s, \quad t \in[0, T), \\
v_{m d d}(t, s, m, m d d) & =0 & & \text { if } m-s=m d d, \quad t \in[0, T) .
\end{aligned}
$$

Proof: This proof is analogous to the derivation of the equation for a lookback option presented in Shreve (2004) (see pages 309-312). Process $e^{-r t} v\left(t, S_{t}, M_{t}, M D D_{t}\right)$ is an $\mathcal{F}_{t}$ measurable martingale under the riskneutral measure $\mathbb{Q}$, which follows from (2). Let us apply the Itô formula to this process:

$$
\begin{aligned}
d e^{-r t} v\left(t, S_{t}, M_{t}, M D D_{t}\right)= & -r e^{-r t} v d t+e^{-r t} d v\left(t, S_{t}, M_{t}, M D D_{t}\right) \\
= & -e^{-r t} r v d t+e^{-r t} v_{t} d t+e^{-r t} v_{s} d S_{t}+ \\
& +e^{-r t} v_{m} d M_{t}+e^{-r t} v_{m d d} d M D D_{t}+e^{-r t} \frac{1}{2} v_{s s} d\left\langle S_{t}\right\rangle \\
= & -e^{-r t} r v d t+e^{-r t} v_{t} d t+e^{-r t} r S_{t} v_{s} d t+e^{-r t} \frac{1}{2} \sigma^{2} S_{t}^{2} v_{s s} d t+ \\
& +e^{-r t} \sigma S_{t} v_{s} d W_{t}+e^{-r t} v_{m} d M_{t}+e^{-r t} v_{m d d} d M D D_{t} \\
= & e^{-r t}\left\{-r v+v_{t}+r S_{t} v_{s}+\frac{1}{2} \sigma^{2} S_{t}^{2} v_{s s}\right\} d t+ \\
& +e^{-r t} v_{m} d M_{t}+e^{-r t} v_{m d d} d M D D_{t}+e^{-r t} \sigma S_{t} v_{s} d W_{t} .
\end{aligned}
$$

According to the martingale representation theorem, there exists a process $\Theta_{t}$, such that $d e^{-r t} v=\Theta_{t} d W_{t}$. This condition is consistent with result (6) if and only if

$$
\begin{aligned}
& \int_{0}^{t} e^{-r u}\left\{-r v+v_{t}+r S_{u} v_{s}+\frac{1}{2} \sigma^{2} S_{u}^{2} v_{s s}\right\} d u=0, \quad t \geq 0, \\
& \int_{0}^{t} e^{-r u} v_{m} d M_{u}=0, \quad t \geq 0, \\
& \int_{0}^{t} e^{-r u} v_{m d d} d M D D_{u}=0, \quad t \geq 0,
\end{aligned}
$$

almost surely. The reason is that none of these terms can be expressed as an integral with respect to $W$ because they have finite first variations.

Condition (7) is satisfied if function $v$ solves the following partial differential equation:

$$
v_{t}(t, s, m, m d d)+r s v_{s}(t, s, m, m d d)+\frac{1}{2} \sigma^{2} s^{2} v_{s s}(t, s, m, m d d)=r v(t, s, m, m d d) .
$$

A path of the process $M_{t}$ is constant except for the set $\left\{t \geq 0 ; S_{t}=M_{t}\right\}$. Thus, (8) holds if $v_{m}(t, s, m, m d d)=0$ for $s=m$. A similar argument can be used for (9). A path of $M D D_{t}$ is constant except for the set $\left\{t \geq 0 ; M_{t}-S_{t}=M D D_{t}\right\}$, which leads to condition $v_{m d d}(t, s, m, m d d)=0$ for $m-s=m d d$.

Now we will discuss the case when the asset value drops to zero, that is, when $S_{t}=0$ and $M_{t}=M D D_{t}$. In this case, $S$ will not recover and the maximum drawdown for the entire period will be $M D D_{t}$. Hence, $v(t, s, m, m d d)=e^{-r(T-t)} m d d$ for $s=0$ or $m=m d d$. 
Since $v\left(T, S_{T}, M_{T}, M D D_{T}\right)=M D D_{T}$, the terminal condition for function $v$ is $v(T, s, m, m d d)=m d d$. $\diamond$

The equation in Proposition 2.1 has three spatial variables defined on a domain which is not rectangular. Nonetheless, a forward on the maximum drawdown has a linear scaling property:

$$
v(t, \lambda s, \lambda m, \lambda m d d)=\lambda v(t, s, m, m d d), \quad \lambda>0,
$$

which allows us to reduce the number of spatial variables to two. Moreover, a certain transformation of variables leads to an equation on a rectangular domain. Hence, let us introduce a function $u(t, x, y)$, such that

$$
v(t, s, m, m d d)=s \cdot u\left(t, \log \left(\frac{m}{s}\right), \log \left(\frac{s}{m-m d d}\right)\right)
$$

According to $(10), u$ is a function of two spatial variables $x$ and $y$ on a rectangular domain $[0, \infty) \times[0, \infty)$ :

$$
\begin{aligned}
& x=\log \left(\frac{m}{s}\right), \\
& y=\log \left(\frac{s}{m-m d d}\right) .
\end{aligned}
$$

Note that the function $u$ can be interpreted as the value of a forward on the maximum drawdown relative to the price of the underlying asset $S$. We derive a partial differential equation for $u$ in the following proposition.

Proposition 2.2 Function $u(t, x, y)$, defined in (10), satisfies the following partial differential equation:

$$
\begin{gathered}
u_{t}-\left(r+\frac{1}{2} \sigma^{2}\right) u_{x}+\left(r+\frac{1}{2} \sigma^{2}\right) u_{y}+\frac{1}{2} \sigma^{2} u_{x x}-\sigma^{2} u_{x y}+\frac{1}{2} \sigma^{2} u_{y y}=0 \\
\text { on }(0, T) \times\{(x, y) ; x>0 \& y>0\} .
\end{gathered}
$$

The terminal and the boundary conditions of $u$ are given by:

$$
\begin{array}{rlrl}
u(T, x, y) & =e^{x}-e^{-y}, & \\
u_{x}(t, x, y)=e^{y} u_{y}(t, x, y) & \text { if } x=0, \quad t \in[0, T), \\
u_{y}(t, x, y)=0 & \text { if } y=0, \quad t \in[0, T) .
\end{array}
$$

Proof: Let us express the derivatives of function $v$ in terms of function $u$ :

$$
\begin{aligned}
v_{t} & =s u_{t}, \\
v_{s} & =u+s\left[-\frac{1}{s} u_{x}+\frac{1}{s} u_{y}\right]=u-u_{x}+u_{y}, \\
v_{s s} & =-\frac{1}{s} u_{x}+\frac{1}{s} u_{y}-\left(-\frac{1}{s} u_{x x}+\frac{1}{s} u_{x y}\right)+\left(-\frac{1}{s} u_{x y}+\frac{1}{s} u_{y y}\right) \\
& =-\frac{1}{s} u_{x}+\frac{1}{s} u_{y}+\frac{1}{s} u_{x x}-\frac{2}{s} u_{x y}+\frac{1}{s} u_{y y}, \\
v_{m} & =s\left(\frac{1}{m} u_{x}-\frac{1}{m-m d d} u_{y}\right)=e^{-x} u_{x}-e^{y} u_{y}, \\
v_{m d d} & =s \frac{1}{m-m d d} u_{y}=e^{y} u_{y} .
\end{aligned}
$$

If we substitute $v$ with $s u$ and the corresponding derivatives in equation (4), we have

$$
s u_{t}+r s u-r s u_{x}+r s u_{y}+\frac{1}{2} \sigma^{2} s\left(-u_{x}+u_{x x}+u_{y}+u_{y y}-2 u_{x y}\right)=r s u
$$


or

$$
u_{t}-\left(r+\frac{1}{2} \sigma^{2}\right) u_{x}+\left(r+\frac{1}{2} \sigma^{2}\right) u_{y}+\frac{1}{2} \sigma^{2} u_{x x}-\sigma^{2} u_{x y}+\frac{1}{2} \sigma^{2} u_{y y}=0,
$$

which proves result (11). Similarly, conditions (12) can be obtained by replacing $v$ with $u$ in (5).

In Section 3, we will discuss a numerical method for solving equation (11) with conditions (12). Let us point out three properties of the equation. First, the terminal condition, $u(T, x, y)=e^{x}-e^{-y}$, is inconsistent with the boundary condition for $y=0: u_{y}(t, x, y)=0$. We denote the coefficients at $u_{x x}$, $u_{x y}$, and $u_{y y}$ as $a_{x x}=(1 / 2) \sigma^{2}, a_{x y}=-\sigma^{2}$, and $a_{y y}=(1 / 2) \sigma^{2}$. It holds that

$$
a_{x x} a_{y y}=\frac{1}{4} a_{x y}^{2}
$$

implying that the diffusion matrix with entries $a_{x x},(1 / 2) a_{x y},(1 / 2) a_{x y}$, and $a_{y y}$ is singular. Thus, one can find new spatial variables, so that the equation contains a second derivative with respect to only one variable. However, the domain would not be rectangular after this transformation. Third, the boundary condition $u_{x}(t, x, y)-e^{y} u_{y}(t, x, y)=0$ for $x=0$ is not of Neumann type because the vector $\left(1,-e^{y}\right)$ is not orthogonal to the boundary $x=0$. The impact of these properties on the stability of the numerical method will be discussed in Section 4.

Before we focus on numerical issues, we state further results on a forward on the maximum drawdown. In the standard Black-Scholes framework, a $\Delta$-hedge is the sensitivity of a contract value to the price of the underlying asset. In our case, the $\Delta$-hedge depends on variables $s, m$, and $m d d$ through $x$ and $y$ because

$$
\Delta(t, s, m, m d d)=v_{s}(t, s, m, m d d)=u(t, x, y)-u_{x}(t, x, y)+u_{y}(t, x, y)
$$

according to the definition of $u$. In the following Proposition 2.3, we present probabilistic representations of the delta hedge and of the value function sensitivities to the running maximum and the running maximum drawdown: $v_{m}$ and $v_{m d d}$. Details about these results can be found in Pospisil and Vecer (2008).

We use the following notation: $\tau_{M_{t}}=T \wedge \inf \left\{u \geq t ; S_{u}=M_{t}\right\}$ and $M D D_{\tau_{M_{t}}}^{t}=\max _{u \in\left[t, \tau_{M_{t}}\right]}\left(M_{u}-S_{u}\right)$. We denote by $\tau_{M_{t}}$ the first time after $t$ when $S_{u}$ attains the value of the running maximum $M_{t}$, and by $M D D_{\tau_{M_{t}}}^{t}$ the maximum drawdown on $\left[t, \tau_{M_{t}}\right]$.

Proposition 2.3 Let $v$ be the value function of a forward on the maximum drawdown,

$$
v\left(t, S_{t}, M_{t}, M D D_{t}\right)=e^{-r(T-t)} \mathbb{E}\left[M D D_{T} \mid S_{t}, M_{t}, M D D_{t}\right]
$$

Then

$$
\begin{aligned}
v_{m d d}\left(t, S_{t}, M_{t}, M D D_{t}\right) & =e^{-r(T-t)} \mathbb{Q}_{t}\left[M D D_{T}=M D D_{t}\right] \\
v_{m}\left(t, S_{t}, M_{t}, M D D_{t}\right) & =e^{-r(T-t)} \mathbb{Q}_{t}\left[M D D_{T}=M D D_{\tau_{M_{t}}}^{t}\right] \\
\Delta\left(t, S_{t}, M_{t}, M D D_{t}\right) & =\frac{V_{t}}{S_{t}}-e^{-r(T-t)}\left(\frac{M_{t}}{S_{t}} \mathbb{Q}_{t}\left[M D D_{T}=M D D_{\tau_{M_{t}}}^{t}\right]+\frac{M D D_{t}}{S_{t}} \mathbb{Q}_{t}\left[M D D_{T}=M D D_{t}\right]\right),
\end{aligned}
$$

where $\mathbb{Q}_{t}$ is the conditional risk-neutral probability, given $S_{t}, M_{t}$, and $M D D_{t}$. Process $\Delta\left(t, S_{t}, M_{t}, M D D_{t}\right)$ has the following properties:

(i) if $M_{0}=S_{0}$ and $M D D_{0}=0$, then $\Delta\left(0, S_{0}, M_{0}, M D D_{0}\right)=\frac{V_{0}}{S_{0}}$,

(ii) $\Delta\left(t, S_{t}, M_{t}, M D D_{t}\right)>-1$ for any $t \in[0, T]$,

(iii) if $S_{t}=M_{t}$, then $\Delta_{t}\left(S_{t}, M_{t}, M D D_{t}\right) \geq 0$,

(iv) $\Delta\left(T, S_{T}, M_{T}, M D D_{T}\right)=0$. 
Proof: A proof can be found in Pospisil and Vecer (2008).

According to Proposition 2.3, sensitivity $v_{m d d}$ can be interpreted as the discounted conditional risk-neutral probability that $M D D_{T}$ has been attained on $[0, t]$ and sensitivity $v_{m}$ as the probability that $M D D_{T}$ will be attained on $\left[t, \tau_{M_{t}}\right]$. This interpretation is consistent with the boundary conditions in (5): $v_{m d d}=0$ if $m-s=m d d$ and $v_{m}=0$ if $m=s$.

\section{Numerical Solution of the Partial Differential Equation}

In this section, we present a numerical method for solving the partial differential equation introduced in Proposition 2.2:

$$
u_{t}-\left(r+\frac{1}{2} \sigma^{2}\right) u_{x}+\left(r+\frac{1}{2} \sigma^{2}\right) u_{y}+\frac{1}{2} \sigma^{2} u_{x x}-\sigma^{2} u_{x y}+\frac{1}{2} \sigma^{2} u_{y y}=0 .
$$

Function $u(t, x, y)$ is defined on $[0, T] \times[0, \infty) \times[0, \infty)$. At the time of maturity, we have:

$$
u(T, x, y)=e^{x}-e^{-y} .
$$

The boundary conditions for the equation are given as follows:

$$
\begin{array}{ll}
u_{x}(t, x, y)=e^{y} u_{y}(t, x, y) & \text { if } x=0, \quad t \in[0, T), \\
u_{y}(t, x, y)=0 & \text { if } y=0, \quad t \in[0, T) .
\end{array}
$$

According to Proposition 2.1 and Proposition 2.2, $u$ is the value of a forward on the maximum drawdown relative to the underlying asset price:

$$
u\left(t, \log \left(\frac{M_{t}}{S_{t}}\right), \log \left(\frac{S_{t}}{M_{t}-M D D_{t}}\right)\right)=\frac{V_{t}}{S_{t}} .
$$

At the end of this section we provide numerical examples of solutions to the partial differential equation.

Equation (13) is a two-dimensional convection-diffusion equation on a rectangular domain, which can be solved numerically using an alternating direction implicit method. In this paper, we implement two standard examples of this method, the Peaceman-Rachford and Douglas-Rachford schemes. The questions of stability and convergence of these schemes are discussed in Section 4.

For the sake of notation, we define the following operators:

$$
\begin{aligned}
A_{x} & =-\left(r+\frac{1}{2} \sigma^{2}\right) \frac{\partial}{\partial x}+\frac{1}{2} \sigma^{2} \frac{\partial^{2}}{\partial x^{2}}, \\
A_{y} & =\left(r+\frac{1}{2} \sigma^{2}\right) \frac{\partial}{\partial y}+\frac{1}{2} \sigma^{2} \frac{\partial^{2}}{\partial y^{2}}, \\
A_{x y} & =-\sigma^{2} \frac{\partial^{2}}{\partial x \partial y} .
\end{aligned}
$$

Hence, we can write equation (13) as

$$
u_{t}+A_{x} u+A_{y} u+A_{x y} u=0 .
$$

In order to define a numerical solution to the equation, we need to truncate the spatial domain to a bounded area: $\left\{(x, y) ; 0 \leq x \leq x_{\max }, 0 \leq y \leq y_{\max }\right\}$. Let us introduce a grid of points in the time interval and in the truncated spatial domain:

$$
\begin{aligned}
t_{i} & =i \frac{T}{I}, \quad i=0, \ldots, I \\
x_{k} & =k \frac{x_{\max }}{K}, \quad k=0, \ldots, K, \\
y_{l} & =l \frac{y_{\max }}{L}, \quad l=0, \ldots, L .
\end{aligned}
$$


The steps of this grid are $d t=T / I, d x=x_{\max } / K$, and $d y=y_{\max } / L$. Function $u$ at a point of the grid will be denoted as $u_{k, l}^{i}=u\left(t_{i}, x_{k}, y_{l}\right)$. If we need to refer to the solution at a specific time point, we will use notation $u^{i}=\left(u_{k, l}^{i}\right)_{k, l}$. Furthermore, let symbols $A_{d x}, A_{d y}$, and $A_{d x d y}$ denote second-order approximations to the operators $A_{x}, A_{y}$, and $A_{x y}$.

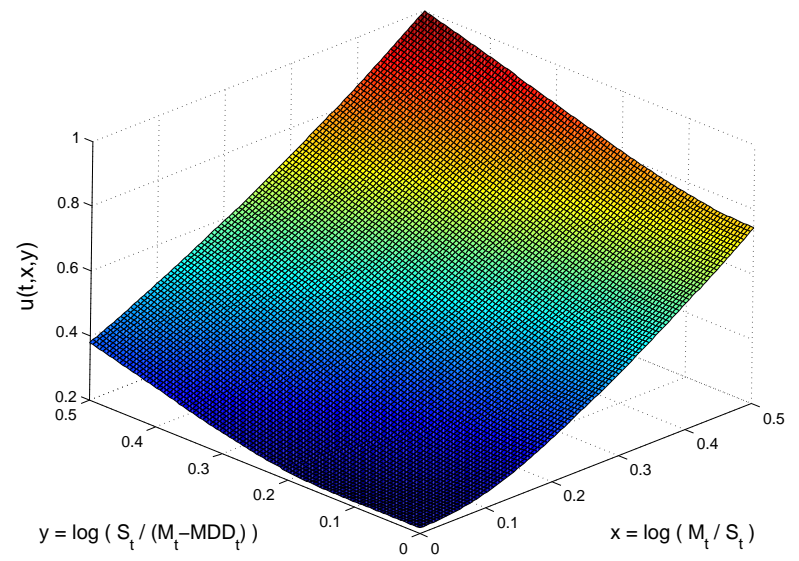

Figure 2: Value of a forward on the maximum drawdown relative to the underlying asset price, $u(t, x, y)$, as a function of $x$ and $y$. Parameters: time to maturity $T-t=1$ year, interest rate $r=4 \%$, volatility $\sigma=19 \%$.

Now we describe an implementation of the alternating direction methods. First, the terminal condition (14) determines the value of the solution at $t_{I}$ :

$$
u_{k, l}^{I}=e^{x_{k}}-e^{-y_{l}} .
$$

As mentioned earlier, we use the Peaceman-Rachford and Douglas-Rachford schemes to obtain $u^{i}$ from $u^{i+1}$, where $i=I-1, I-2, \ldots, 0$. A derivation of these methods can be found, for example, in Strikwerda (1989) (Chapter 7.3) or Duffy (2006) (Chapter 19). The Peaceman-Rachford scheme is defined as:

$$
\begin{array}{ll}
\left(\mathbb{I}-\frac{d t}{2} A_{d x}\right) u^{i+1 / 2} & =\left(\mathbb{I}+\frac{d t}{2} A_{d y}\right) u^{i+1}+\frac{d t}{2} A_{d x d y} u^{i+1}, \\
\left(\mathbb{I}-\frac{d t}{2} A_{d y}\right) u^{i} & =\left(\mathbb{I}+\frac{d t}{2} A_{d x}\right) u^{i+1 / 2}+\frac{d t}{2} A_{d x d y} u^{i+1 / 2}
\end{array}
$$

where $\mathbb{I}$ denotes the identity operator. Auxiliary function $u^{i+1 / 2}$ links equations (18) and (19). The DouglasRachford scheme is defined in the following way:

$$
\begin{array}{ll}
\left(\mathbb{I}-d t A_{d x}\right) u^{i+1 / 2} & =\left(\mathbb{I}+d t A_{d y}\right) u^{i+1}+d t A_{d x d y} u^{i+1}, \\
\left(\mathbb{I}-d t A_{d y}\right) u^{i} & =u^{i+1 / 2}-d t A_{d y} u^{i+1} .
\end{array}
$$

Note that both schemes have similar structures. In the first step, (18) or (20), we calculate $u^{i+1 / 2}$ using $u^{i+1}$. This step is implicit in direction $x$, therefore we need to specify boundary conditions for $x=0$ and $x=x_{\max }$. Values at $x=0$ can be obtained by discretization of (15), where we use values at time $i+1$ for direction $y$ and at time $i+1 / 2$ for direction $x$ :

$$
e^{y_{l}} \frac{u_{0, l+1}^{i+1}-u_{0, l-1}^{i+1}}{2 d y}=\frac{-3 u_{0, l}^{i+1 / 2}+4 u_{1, l}^{i+1 / 2}-u_{2, l}^{i+1 / 2}}{2 d x} .
$$




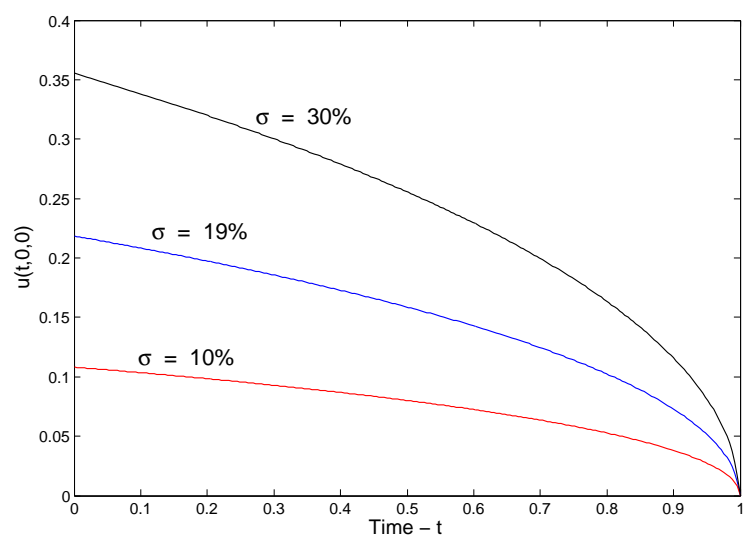

Figure 3: Value of a forward on the maximum drawdown relative to the underlying asset price, $u(t, 0,0)$, as a function of $t$. Parameters: time of maturity $T=1$ year, interest rate $r=4 \%$. Volatilities: $\sigma=10 \%, \sigma=19 \%$ (historical volatility of S\&P 500 from March 26, 2007 to March 20, 2008), and $\sigma=30 \%$.

If $x=x_{\max }$, then $s$ is small if compared to $m$. Let us assume that in this case, condition $v(t, s, m, m d d)=$ $e^{-r(T-t)} m d d$ in (5) approximately holds. Thus, $u$ grows at the rate $e^{-r\left(T-t_{i}+d t / 2\right)} e^{x_{\max }}$ in the direction of $x$ when $x=x_{\max }$, which gives us the following second-order approximation:

$$
u_{K, l}^{i}=\frac{1}{3}\left(4 u_{K-1, l}^{i}-u_{K-2, l}^{i}+2 d x e^{-r\left(T-t_{i}+d t / 2\right)} e^{x_{K}}\right), \quad l=1, \ldots, L .
$$

As a result, we can solve the system of equations (18) or (20) with conditions (22) and (23) to obtain $u^{i+1 / 2}$.

In the second step, defined by equations (19) or (21), we use $u^{i+1 / 2}$ to calculate $u^{i}$. This step is implicit in the direction of $y$. Thus, we need to approximate boundary conditions for $y=0$ and $y=y_{\max }$. A second-order discretization of (16) gives us an equation for values at $y=0$ :

$$
\frac{-3 u_{k, 0}^{i}+4 u_{k, 1}^{i}-u_{k, 2}^{i}}{2 d y}=0 .
$$

If $y=y_{\max }$, then $m-m d d$ is small if compared to $s$. Let us assume that condition $v(t, s, m, m d d)=$ $e^{-r(T-t)} m d d$ for $m-m d d=0$ holds in this case and $u$ is approximately $e^{-r(T-t)}\left(e^{x}-e^{-y_{\max }}\right)$. Hence,

$$
u_{k, L}^{i}=e^{-r\left(T-t_{i}\right)}\left(e^{x_{k}}-e^{-y_{L}}\right), \quad k=1, \ldots, K .
$$

Equations (19) or (21) together with (24) and (25) allow us to calculate $u^{i, k, l}$ for $k \geq 1$. To complete this step, we need to determine values $u_{0, l}^{i}, l=1, \ldots, L$. This can be done by a second-order discretization of $u_{x}(t, x, y)=e^{y} u_{y}(t, x, y)$ on boundary $x=0$ :

$$
\begin{aligned}
\frac{-3 u_{0,0}^{i}+4 u_{0,1}^{i}-u_{0,2}^{i}}{2 d y} & =0, \\
\frac{u_{0, l+1}^{i}-u_{0, l-1}^{i}}{2 d y} & =e^{-y_{l}} \frac{-3 u_{0, l}^{i}+4 u_{1, l}^{i}-u_{2, l}^{i}}{2 d x}, \quad l=1, \ldots, L-1, \\
\frac{3 u_{0, L}^{i}-4 u_{0, L-1}^{i}+u_{0, L-2}^{i}}{2 d y} & =e^{-y_{l}} \frac{-3 u_{0, L}^{i}+4 u_{1, L}^{i}-u_{2, L}^{i}}{2 d x} .
\end{aligned}
$$



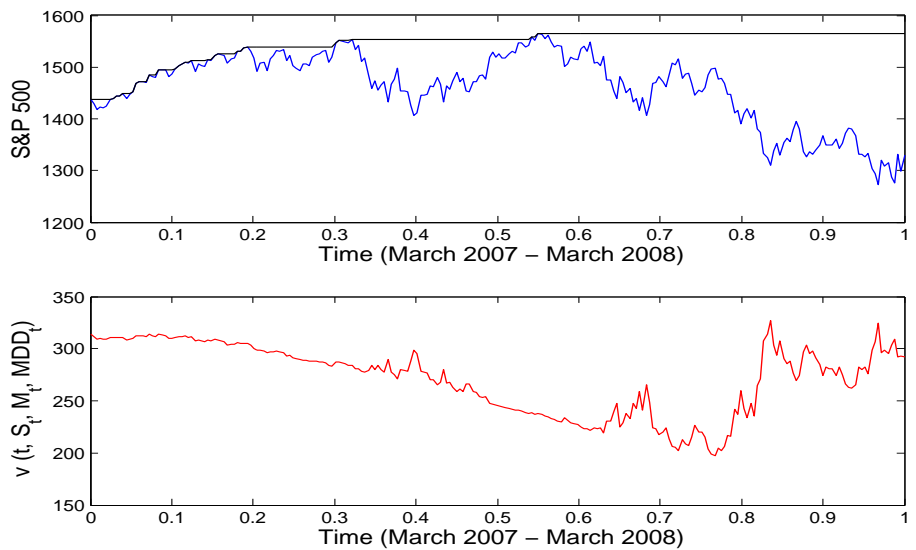

Figure 4: The path in the upper figure represents the historical values of S\&P 500 from March 26, 2007 to March 20, 2008. The lower figure shows the corresponding path of $v\left(t, S_{t}, M_{t}, M D D_{t}\right)$, the price of a forward on the maximum drawdown of S\&P 500, where March 26, 2007 is the beginning of the contract and March 20, 2008 is the day of maturity. We use the following parameters: interest rate $4 \%$ and volatility $19 \%$.

The first equation follows from the fact that $u_{y}=0$ for $x=y=0$.

Now all values $\left(u_{k, l}^{i}\right)_{k, l}$ are available. By repeating this procedure for $i=I-1, I-2, \ldots, 0$, we obtain $u^{i}$ at all time points. The price of a forward on the maximum drawdown at time $t=0$ can be approximated as:

$$
v\left(0, S_{0}, M_{0}, M D D_{0}\right) \approx S_{0} u_{0,0}^{0} .
$$

Examples of numerical solutions to equation (13) are shown in Figures 3 - 6. All of the results were obtained using the Douglas-Rachford alternating direction method. Figure 3 displays $u(t, x, y)$ as a function of $x$ and $y$. Recall that $u$ represents the value of a forward on the maximum drawdown relative to the price of the underlying asset $S$. The time to maturity is $T-t=1$ year, interest rate $r=4 \%$, and volatility $\sigma=19 \%$. In Figure 3, we have plotted $u(t, 0,0)$ as a function of $t$, for three different volatilities: $\sigma=10 \%, \sigma=19 \%$, and $\sigma=30 \%$. The parameters for this figure are $T=1$ year and $r=4 \%$. Figure 4 shows a path of the process $v\left(t, S_{t}, M_{t}, M D D_{t}\right)$, where $S$ represents S\&P 500 index from March 26, 2007 to March 20, 2008. The empirical annual volatility of $S$ over this period was $19 \%$.

Figure 5 shows the $\Delta$-hedge of a forward on the maximum drawdown as a function of $x$ and $y, \Delta(t, x, y)=$ $u(t, x, y)-u_{x}(t, x, y)+u_{y}(t, x, y)$. As in Figure 3, the time to maturity is $T-t=1$ year, interest rate $r=4 \%$, and volatility $\sigma=19 \%$. Finally, Figure 6 shows a path of the process $\Delta\left(t, S_{t}, M_{t}, M D D_{t}\right)$ for S\&P 500 from March 26, 2007 to March 20, 2008.

\section{Stability and Convergence of the Numerical Solution}

In this section, we discuss stability and convergence of the numerical methods introduced in Section 3 . The stability analysis has two components. First, we determine when the methods are stable if they are applied to a terminal-value problem with periodic boundary conditions. This part can be accomplished using known theoretical results. Second, we examine the influence of boundary conditions (15) and (16) on the stability 


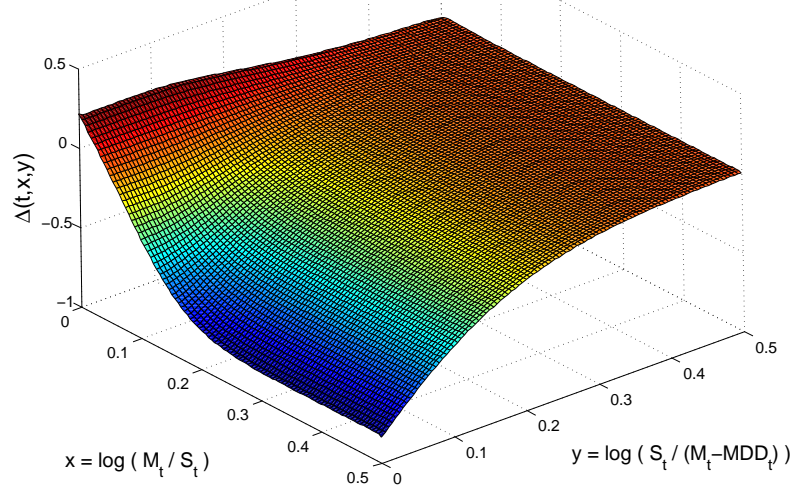

Figure 5: $\Delta$-hedge of a forward on the maximum drawdown: $\Delta(t, x, y)=u-u_{x}+u_{y}$. Parameters: time to maturity $T-t=1$ year, interest rate $r=4 \%$, and volatility $\sigma=19 \%$.

results from the first part. The conclusion we draw about the stability of the boundary conditions is based on numerical experiments.

We first analyze the stability of the Peaceman-Rachford and Douglas-Rachford schemes for (13), assuming that the boundary conditions are periodic. In this case, we can use the von Neumann analysis to establish the conditions of stability. This approach was described in Strikwerda (1989) (Chapter 2.2). The von Neumann analysis is based on calculating the amplification factor of a scheme, $g$, and deriving conditions under which $|g| \leq 1$.

Recall that $I$ is the number of time steps in interval $[0, T]$ and $K$ and $L$ the numbers of steps in $\left[0, x_{\max }\right]$ and $\left[0, y_{\max }\right]$, respectively. For the simplicity of notation, we assume that $x_{\max }=y_{\max }$ and $K=L$. The amplification factor of the Peaceman-Rachford scheme (18) and (19) is:

$$
\begin{aligned}
& |g(\theta, \phi)|^{2}= \\
& =\frac{\left[\left(1-4 a \sin ^{2}(\theta / 2)+2 a \sin \theta \sin \phi\right)^{2}+4 b^{2} \sin ^{2} \theta\right]\left[\left(1-4 a \sin ^{2}(\phi / 2)+2 a \sin \theta \sin \phi\right)^{2}+4 b^{2} \sin ^{2} \phi\right]}{\left[\left(1+4 a \sin ^{2}(\theta / 2)\right)^{2}+4 b^{2} \sin ^{2} \theta\right]\left[\left(1+4 a \sin ^{2}(\phi / 2)\right)^{2}+4 b^{2} \sin ^{2} \phi\right]},
\end{aligned}
$$

where

$$
\begin{aligned}
a & =\frac{1}{2} \frac{T}{I} \frac{K^{2}}{x_{\max }^{2}} \frac{\sigma^{2}}{2}, \\
b & =\frac{1}{2} \frac{T}{I} \frac{K}{2 x_{\max }}\left(r+\frac{\sigma^{2}}{2}\right) .
\end{aligned}
$$

One can show that a sufficient condition for the amplification factor to be bounded by $1,|g(\theta, \phi)| \leq 1$, is $a \leq 1$, or:

$$
I \geq \frac{K^{2} \sigma^{2} T}{4 x_{\max }^{2}}
$$

Thus, the Peaceman-Rachford scheme is stable if the number of steps in the time interval, $I$, and in the spatial domain, $K$, satisfy inequality (27). This condition is a consequence of the cross-derivative term in equation (13), represented by $2 a \sin \theta \sin \phi$ in the formula for the amplification factor. In the absence of this term, the scheme would be unconditionally stable. 

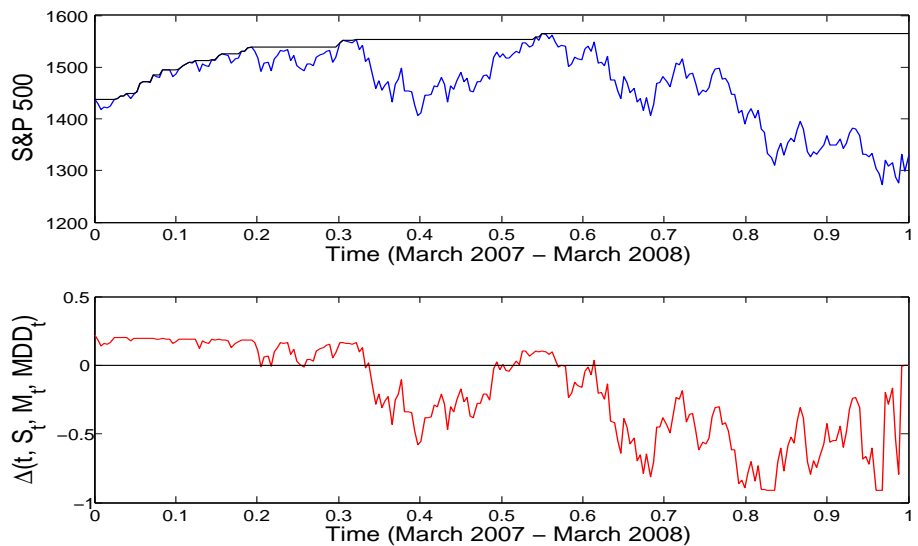

Figure 6: In the upper figure, we have plotted the evolution of S\&P 500 from March 26, 2007 to March 20, 2008. The lower figure shows the corresponding path of $\Delta\left(t, S_{t}, M_{t}, M D D_{t}\right)$, the $\Delta$-hedge of a forward on the maximum drawdown. S\&P 500 is the underlying asset, March 26, 2007 is the beginning of the contract and March 20, 2008 is the day of maturity. We use the following parameters: interest rate $r=4 \%$ and volatility $\sigma=19 \%$. Note that process $\Delta\left(t, S_{t}, M_{t}, M D D_{t}\right)$ exhibits the properties listed in Proposition 2.3.

The stability of the Douglas-Rachford scheme (20) and (21) was analyzed by in't Hout and Welfert (2007), who proved that this scheme, including the cross-derivative term, is unconditionally stable.

Let us discuss the impact of boundary conditions (15) and (16) and their finite difference approximations on the stability of the numerical methods. According to the numerical experiments we carried out, the boundary conditions does not change the stability results from the first part of the analysis. In other words, even after including conditions (15) and (16), the Peaceman-Rachford scheme is stable if $I$ and $K$ satisfy (27) and the Douglas-Rachford scheme is unconditionally stable.

The remaining issue we need to address is the convergence of the numerical methods to the true value of a forward on the maximum drawdown. We focus only on the Douglas-Rachford scheme due to its unconditional stability. According to Strikwerda (1989), this scheme is first-order accurate in time and second-order accurate in space. As pointed out in Section 1, no analytical expression for the expected maximum drawdown is known. However, if we change the terminal condition for the equation (13) to

$$
u_{L B}(T, x, y)=e^{x}-1,
$$

we have:

$$
\begin{aligned}
v_{L B}\left(T, S_{T}, M_{T}, M D D_{T}\right) & =S_{T} u_{L B}\left(t, \log \left(\frac{M_{T}}{S_{T}}\right), \log \left(\frac{S_{T}}{M_{T}-M D D_{T}}\right)\right)=S_{T}\left(\exp \left(\log \left(\frac{M_{T}}{S_{T}}\right)\right)-1\right) \\
& =M_{T}-S_{T} .
\end{aligned}
$$

Note that this is the payoff of a floating strike lookback put option. Thus, the numerical methods introduced in Section 3 with terminal condition (28) can be used to price this lookback option. Since there is a formula for the value of the option, we can compare the results obtained from the numerical method with the true value of the option.

Results of this convergence study are summarized in Table 1. The relative pricing errors for the finest mesh 


\begin{tabular}{|c|c|c|c|c|}
\hline \multicolumn{5}{|c|}{ Douglas-Rachford Method } \\
\hline $\bar{I}$ & $\mathrm{~K}, \mathrm{~L}$ & $\sigma=10 \%$ & $\sigma=19 \%$ & $\sigma=30 \%$ \\
\hline 100 & 100 & 83.9095 & 184.8068 & 316.9884 \\
\hline 400 & 200 & 83.9591 & 184.9738 & 317.2532 \\
\hline 900 & 300 & 83.9684 & 185.0044 & 317.2989 \\
\hline \multicolumn{2}{|c|}{ Analytical price } & 83.9759 & 185.0284 & 317.3161 \\
\hline
\end{tabular}

Table 1: Convergence of the Douglas-Rachford method introduced in Section 3 for a lookback put option. Parameters: $S_{0}=1329.5$ (S\&P 500 on March 20, 2008), time to maturity $T=1$ year, $r=4 \%$, and $x_{\max }=y_{\max }=0.6$. We study the convergence for three different levels of volatilities: $\sigma=10 \%, \sigma=19 \%$ (historical volatility of S\&P 500 from March 26, 2007 to March 20, 2008), and $\sigma=30 \%$. I denotes the number of time steps, while $K$ and $L$ are the numbers of steps in the spatial domain.

in the table, $I=900$ and $K=L=300$, are: $0.0089 \%$ (for $\sigma=10 \%$ ), $0.0130 \%$ (for $\sigma=19 \%$ ), and $0.0054 \%$ (for $\sigma=30 \%$ ).

\section{Conclusion}

In this paper, we study a forward on the maximum drawdown. This contract can serve as insurance against adverse market movements and its price as a risk measure in portfolio management. Since there is no known analytical formula for the expected maximum drawdown, we propose a partial differential equation approach to price the forward. First, we derive a three-dimensional partial differential equation for the forward in the Black-Scholes framework, and then we reduce its dimensionality to two using the linear scaling argument.

We apply two standard alternating direction implicit methods to solve the equation - the Peaceman-Rachford scheme and the Douglas-Rachford scheme. There are several issues related to the equation that requires attention. One of them is that a boundary condition is neither Dirichlet nor Neumann type. Finally, we discuss the stability and the convergence of the schemes.

\section{References}

[1] Chekhlov, A., S. Uryasev, and M. Zabarankin (2005): Drawdown Measure in Portfolio Optimization, International Journal of Theoretical and Applied Finance, Vol. 8, No. 1, 13-58.

[2] Cvitanic, J. and I. Karatzas (1995): On Portfolio Optimization under Drawdown Constraints, IMA Lecture Notes in Mathematics \& Applications, 65, 77-88.

[3] Douglas, J. and H. H. Rachford (1956): On the Numerical Solution of Heat Conduction Problems in Two and Three Space Variables, Transactions of the American Mathematical Society, Vol. 82, No. 2, 421-439.

[4] Duffy, D. J. (2006): Finite Difference Methods in Financial Engineering, Wiley Finance.

[5] Grossman, S. J. And Z. Zhou (1993): Optimal Investment Strategies for Controlling Drawdowns, Mathematical Finance, Vol. 3, No. 3, 241-276.

[6] in't Hout, K. J. And B. D. Welfert (2007): Stability of ADI Schemes Applied to ConvectionDiffusion Equations with Mixed Derivative Terms, Applied Numerical Mathematics, Vol. 57, No. 1, $19-35$. 
[7] Hundsdorfer, W. H. and J. G. Verwer (1989): Stability and Convergence of the PeacemanRachford ADI Method for Initial Boundary Value Problems, Mathematics of Computation, Vol. 53, No. $187,81-101$.

[8] Magdon-Ismail, M., A. Atiya, A. Pratap, and Y. Abu-Mostafa (2004): On the Maximum Drawdown of a Brownian Motion, Journal of Applied Probability, Vol. 41, No. 1.

[9] Peaceman, D. W. and H. H. Rachford (1955): The Numerical Solution of Parabolic and Elliptic Differential Equations, Journal of the Society for Industrial and Applied Mathematics, Vol. 3, No. 1, $28-41$.

[10] Pospisil, L. And J. Vecer (2008): Portfolio Sensitivities to the Changes in the Maximum and the Maximum Drawdown, Preprint.

[11] Shreve, S. (2004): Stochastic Calculus for Finance II, Springer Verlag.

[12] Strikwerda, J. C. (1989): Finite Difference Schemes and Partial Differential Equations, Chapman 83 Hall.

[13] Vaz de Melo Mendes, B. and V. Ratton Brandi (2004):, Modeling Drawdowns and Drawups in Financial Markets, The Journal of Risk, Vol. 6, No. 3.

[14] Vecer, J. (2006): Maximum Drawdown and Directional Trading, Risk, Vol. 19, No. 12, 88-92. 